\title{
An activated Ras protein alters cell adhesion by dephosphorylating Dictyostelium DdCAD-1
}

\begin{abstract}
Correspondence
Gerald Weeks

gerwee@interchange.ubc.ca
\end{abstract}

Received 23 November 2005

Accepted 26 January 2006

\author{
David M. Secko, ${ }^{1}$ Chi-Hung Siu, ${ }^{2}$ George B. Spiegelman ${ }^{1}$ \\ and Gerald Weeks ${ }^{1}$
}

\author{
${ }^{1}$ Department of Microbiology and Immunology, University of British Columbia, Life Sciences \\ Centre, 2350 Health Sciences Mall, Vancouver, British Columbia V6T 1Z4, Canada \\ ${ }^{2}$ Banting and Best Department of Medical Research, and Department of Biochemistry, \\ University of Toronto, Toronto, Ontario M5G 1L6, Canada
}

\begin{abstract}
RasG-regulated signal transduction has been linked to a variety of growth-specific processes and appears to also play a role in the early development of Dictyostelium discoideum. In an attempt to uncover some of the molecular components involved in Ras-mediated signalling, several proteins have been described previously, including the cell adhesion molecule DdCAD-1, whose phosphorylation state was affected by the expression of the constitutively activated RasG, RasG(G12T). Here it has been shown that a cadA null strain lacks the phosphoproteins that were tentatively identified as DdCAD-1, confirming its previous designation. Further investigation revealed that cells expressing RasG(G12T) exhibited increased cell-cell cohesion, concomitant with reduced levels of DdCAD-1 phosphorylation. This increased cohesion was DdCAD-1-dependent and was correlated with increased localization of DdCAD-1 at the cell surface. DdCAD-1 phosphorylation was also found to decrease during Dictyostelium aggregation. These results revealed a possible role for protein phosphorylation in regulating DdCAD-1-mediated cell adhesion during early development. In addition, the levels of DdCAD-1 protein were substantially reduced in a ras $G$ null cell line. These results indicate that RasG affects both the expression and dephosphorylation of DdCAD-1 during early development.
\end{abstract}

\section{INTRODUCTION}

Cell-to-cell adhesion is an important mechanism in multicellular organisms for maintaining cell shape and tissue structure, but it is also a point of integration between cell contact and intercellular signalling (Juliano, 2002; Coates \& Harwood, 2001). In mammalian cells, cell adhesion receptors, such as cadherins, integrins and selectins, have been linked to the regulation of intercellular signalling pathways (Juliano, 2002). However, there are still many gaps in our understanding of the relationship between cell signalling and cell adhesion and, in particular, the mechanisms by which signalling molecules, such as Ras, affect and are affected by cell adhesion.

The importance of adhesion during Dictyostelium development has been long recognized (Gerisch, 1968). These solitary amoebae feed on bacteria during the vegetative phase but, upon nutrient deprivation, up to $10^{5}$ cells aggregate, spatially organize and differentiate into multiple cell types (Kessin, 2001). The adhesion protein DdCAD-1 (previously known as contact site B or gp24) is one of the first proteins to be expressed at the cell surface at the onset of starvation (Beug et al., 1973; Yang et al., 1997) and it has been implicated in mediating initial side-to-side contacts during early aggregation (Beug et al., 1973; Sesaki \& Siu, 1996). A second adhesion molecule, termed gp 80 or contact site A, is induced by cAMP as development proceeds (Muller \& Gerisch, 1978; Faix et al., 1992) and mediates a second type of contact in the developing stream (Beug et al., 1973; Choi \& Siu, 1987). gp80 allows tight adhesion between cells following on from the initial adhesive event mediated by DdCAD-1. In addition to the differences in developmental timing, gp80 and DdCAD-1 mediated contacts can also be distinguished by their sensitivity to EDTA, since DdCAD-1 forms EDTA-sensitive contacts, while gp80 forms EDTAinsensitive contacts (Coates \& Harwood, 2001).

Dictyostelium has a large number of Ras proteins, of which six (RasG, RasC, RasD, RasB, RasS and Rap1) have been partially characterized (Weeks \& Spiegelman, 2003). A role for the RasG signalling pathway in early development has been previously suggested by the findings that the expression of a constitutively activated version of RasG, RasG(G12T), blocks development (Khosla et al., 1996) and the initiation of development of ras $G$ null cells is delayed (Tuxworth et al., 1997). This effect appears to be at least partially due to the role of RasG in regulating cAMP production, with both cells expressing RasG(G12T) and lacking rasG showing defects in this essential process (Khosla et al., 1996; P. Bolourani \& 
G. Weeks, unpublished observations). In addition, RasG is activated in response to cAMP stimulation (Kae et al., 2004) and it influences the expression of the discoidin gene (Secko et al., 2001), a known molecular marker of the transition from growth to development (Zeng et al., 2000; Primpke et al., 2000). Recently, we reported the identification of proteins whose phosphorylation state was altered by the ectopic expression of RasG(G12T) (Secko et al., 2004). One of the phosphoproteins identified was DdCAD-1. This identification raises an important question: does DdCAD-1 phosphorylation regulate DdCAD-1-mediated cell adhesion? To address this question, we have analysed DdCAD-1-mediated adhesion in a strain expressing RasG(G12T). These studies suggest that the dephosphorylation of DdCAD-1 is affected by the expression of RasG and thereby influences Dictyostelium cell adhesion.

\section{METHODS}

Dictyostelium strains and growth conditions. Dictyostelium discoideum strains were grown in HL5 medium (Watts \& Ashworth, $1970)$ in rotary-agitated suspension (175 r.p.m.) at $22^{\circ} \mathrm{C}$. The generation of the Ax2::MB, Ax2::MB-rasG(G12T), rasG null (IR17) and cadA null strains have been described previously (Secko et al., 2004; Tuxworth et al., 1997; Wong et al., 2002). Ax2::MB and Ax2 : : MB-rasG(G12T) transformants were maintained and grown in $10 \mu \mathrm{g} \mathrm{G} 418 \mathrm{ml}^{-1}, 5 \mu \mathrm{g}$ blasticidin $\mathrm{S} \mathrm{ml}^{-1}$ and $5 \mu \mathrm{g} \mathrm{ml}$ tetracycline $\mathrm{ml}^{-1}$. For RasG(G12T) induction, cells were centrifuged at $700 \boldsymbol{g}$ for 4 min, washed three times in HL5 medium and then resuspended in HL5 medium containing no tetracycline (Secko et al., 2004). Strains were incubated in rotary-agitated suspension ( 175 r.p.m.) at $22^{\circ} \mathrm{C}$ during the induction period.

Electrophoretic and immunoblot analysis. One-dimensional (1D) SDS-PAGE and immunoblot analysis of fractionated proteins was performed as reported previously (Secko et al., 2001), except that $180 \times 160 \times 1 \mathrm{~mm}$ SDS-polyacrylamide gels were used. These gels were run at a constant current of $25 \mathrm{~mA}$ per gel for $3 \mathrm{~h}$ with cooling. Immunoblots were scanned using a UMAX-II scanner and densitometry was then performed using GeneQuant Analysis software (Molecular Dynamics). Two-dimensional (2D) electrophoresis and immunoblot analysis were performed as reported previously (Secko et al., 2004), except that $7 \mathrm{~cm}, \mathrm{pH} 3-10$, ready-to-use Immobiline DryStrips were rehydrated overnight in the presence of $100 \mu \mathrm{g}$ total protein solution. Antibodies used were as follows: phosphothreonine polyclonal antibody ( $1: 1000$ dilution) (Cell Signalling Technology, cat. \#9381), DdCAD-1 specific polyclonal antibody (1:3000 dilution) (Sesaki \& Siu, 1996) and RasG- and RasC-specific polyclonal antibodies (1:2000 dilution) (Khosla et al., 1994; Lim et al., 2001).

Cell cohesion assays. Cell cohesion assays were performed as described by Wong et al. (2002). To determine developmental cell cohesion, vegetative cells were centrifuged at $700 \mathrm{~g}$ for $4 \mathrm{~min}$, washed in $\mathrm{KK} 2$, resuspended in KK2 at $2 \times 10^{7} \mathrm{cells} \mathrm{ml}^{-1}$ and starved in rotary-agitated suspension ( 175 r.p.m.) at $22^{\circ} \mathrm{C}$ for 4 h. The cell suspension was diluted to a density of approximately $2.5 \times 10^{6}$ cells $\mathrm{ml}^{-1}$ and aggregates were dispersed by vigorously vortexing for $15 \mathrm{~s}$. Aggregates were allowed to reform while rotating on a platform shaker at 180 r.p.m. at room temperature. At the indicated times, the number of non-aggregated cells, including singlets and doublets, were scored using a haemocytometer and the number of aggregating cells was determined by subtracting this number from the total number of cells and was expressed as a percentage of the total.
Vegetative cell cohesion was measured following incubation in HL5 medium alone or HL5 medium supplemented with either $10 \mathrm{mM}$ EDTA or $50 \mu \mathrm{g}$ DdCAD-1-specific antibodies $\mathrm{ml}^{-1}$ (Sesaki \& Siu, 1996). After the indicated times, cells were placed in Nunc tissue culture dishes, viewed using an Olympus CK inverted microscope and photographed with a DAGE-100 CCD camera (Dage Corp.).

Indirect immunofluorescence. Cells were deposited on No. 1 glass cover slips (VWR Scientific) and allowed to adhere for $10 \mathrm{~min}$ in HL5 medium. Once adhered, the medium was removed and the cells were gently washed with KK2 to remove the remaining medium. Cells were fixed in $4 \%$ formaldehyde in KK2 for $15 \mathrm{~min}$ at room temperature. When cells were to be permeabilized, the formaldehyde was removed and cold methanol $\left(-20^{\circ} \mathrm{C}\right)$ containing $1 \%$ formaldehyde was added for 5 min (Fukui et al., 1987). Non-specific binding was blocked with $1 \%(\mathrm{w} / \mathrm{v}) \mathrm{BSA}$ in PBS for $10 \mathrm{~min}$, prior to incubation with the DdCAD-1 antiserum $(1: 400$ dilution in PBS containing $1 \%$ BSA) for $1 \mathrm{~h}$ (Sesaki \& Siu, 1996). Samples were washed three times in PBS containing 0.05\% Tween 20, stained with $1: 200$ FITC-conjugated goat anti-rabbit IgG (Jackson Laboratories) for $1 \mathrm{~h}$ and then washed three times in PBS containing 0.05\% Tween 20. The cover slips were mounted in Prolong Antifade mounting medium (Molecular Probes) on Premium microscope slides (Fisher) and images were acquired using a Zeiss Axioplan fluorescent microscope equipped with $\times 60$ objective lenses using a DVC camera (Diagnostic Instruments) and Northern Eclipse imaging software. The specificity of DdCAD-1 antiserum for DdCAD-1 has been confirmed previously (Sesaki \& Siu, 1996).

\section{RESULTS}

\section{DdCAD-1 is a RasG-regulated phosphoprotein}

MS/MS protein sequencing in combination with $2 \mathrm{D}$ immunoblot analysis and an anti-phosphothreonine antibody previously identified three proteins with amino acid sequences identical to DdCAD-1 (Secko et al., 2004). The relative levels of these three phosphoproteins was reduced in response to the ectopic expression of constitutively activated RasG, RasG(G12T), from a tetracycline-repressible promoter (Secko et al., 2004). The availability of a strain in which the DdCAD-1-encoding gene ( $\operatorname{cadA}$ ) had been disrupted (Wong et al., 2002) provided an opportunity to confirm that the three phosphoproteins were in fact DdCAD-1. Total protein samples from the $c a d A$ null strain $\left(\mathrm{cadA}^{-}\right)$, along with a control $A \times 2$ strain $(\mathrm{Ax} 2:: \mathrm{MB})$ and a strain expressing $\operatorname{RasG}(\mathrm{G} 12 \mathrm{~T})[\mathrm{Ax} 2:: \mathrm{MB}-\operatorname{ras} G(\mathrm{G} 12 \mathrm{~T})]$, were subjected to $2 \mathrm{D}$ immunoblot analysis. The three phosphothreonine-reacting spots that had been characterized as DdCAD-1 were all present in Ax2::MB (Secko et al., 2004), but were absent from the cadA null strain (Fig. 1A). The corresponding silver-stained protein spots were also absent from the cadA null strain (Fig. 1B). Together these results support the conclusion that the phosphothreonine-reacting spots were phosphorylated DdCAD-1.

As previously reported, ectopic expression of RasG(G12T) decreased the overall phosphorylation level of the DdCAD-1 protein detected with the phosphothreonine antibody. This reduction could be interpreted in two ways: (i) as changes in activity of kinases or phosphatases in the cell that result in altered phosphorylation, or (ii) changes in the total protein 
(A)

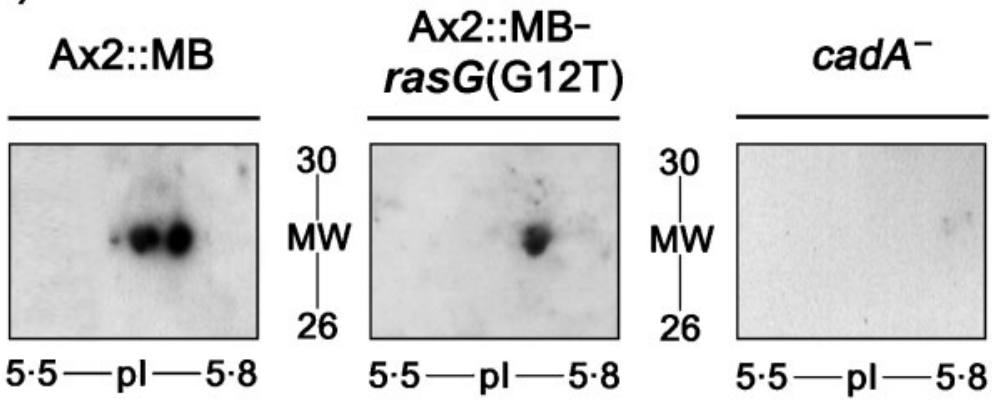

(B)

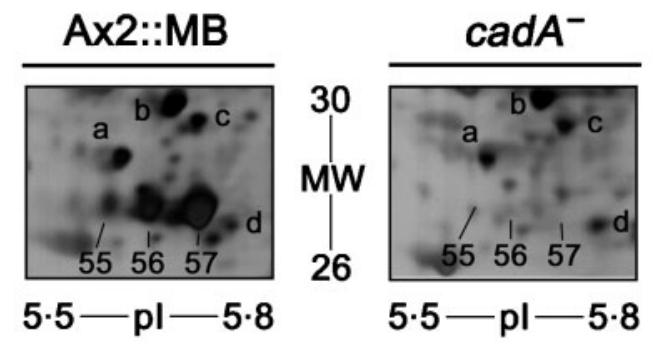

(C)

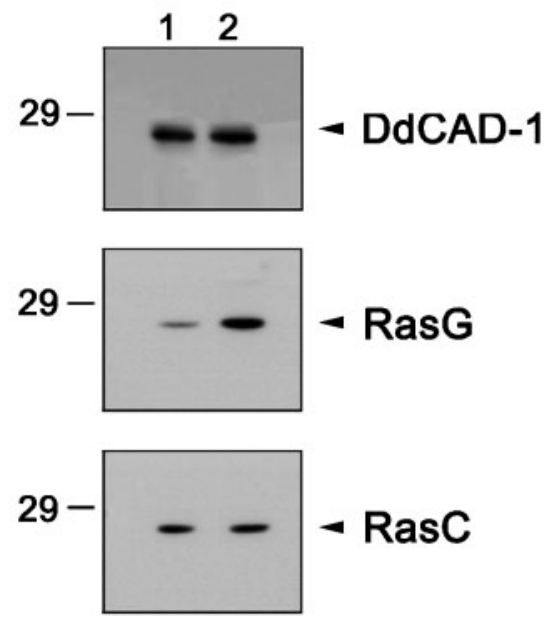

Fig. 1. Phosphorylated and total levels of DdCAD-1. (A, B) Total protein $(100 \mu \mathrm{g})$ from $A \times 2:: M B, A \times 2:: M B-r a s G(G 12 T)$ and cadA null cells was fractionated by $2 \mathrm{D}$ electrophoresis and blotted membranes were probed with anti-phosphothreonine antibody to detect phosphoproteins and silver-stained to detect total protein. The section of the $2 \mathrm{D}$ immunoblots and silver-stained 2D gels known to contain the s55, s56 and s57 immunospots, identified previously as DdCAD-1, is shown. Additional silver-stained spots ( $a, b, c, d)$ are labelled for reference in (B). Molecular mass $(\mathrm{kDa})$ markers and $\mathrm{pl}$ ranges are indicated. (C) Total protein from Ax2::MB (1) and Ax2::MB-rasG(G12T) (2) cells $3 \mathrm{~h}$ after removal of tetracycline was fractionated by 1D electrophoresis. DdCAD1, RasG and RasC proteins levels were detected by immunoblotting with specific antibodies to each of the proteins as described in Methods. Molecular mass markers $(\mathrm{kDa})$ are indicated.

level of these targets. To distinguish between these possibilities, $\mathrm{Ax} 2: \mathrm{MB}$ and $\mathrm{Ax} 2: \mathrm{MB}-\operatorname{ras} G(\mathrm{G} 12 \mathrm{~T})$ cells were sampled after $3 \mathrm{~h}$ induction and lysed directly in SDS-PAGE lysis buffer. Ten micrograms of protein was fractionated by 1D SDS-PAGE, transferred to a PVDF membrane and subjected to immunoblot analysis (Fig. 1C). The levels of DdCAD-1, detected with a DdCAD-1-specific antibody (Sesaki \& Siu, 1996), remained constant. The level of RasC, a protein that was not expected to be affected by RasG(G12T) induction, also showed no change (Fig. 1C) and, as anticipated from a previous study (Secko et al., 2004), the level of RasG protein increased (Fig. 1C). These results indicate that the total level of DdCAD-1 did not change during $\operatorname{RasG}(\mathrm{G} 12 \mathrm{~T})$ induction and confirmed that the observed changes in the phosphothreonine immunoblots were due to decreases in protein phosphorylation.

\section{RasG(G12T) expression results in vegetative cell clumping}

Vegetative Dictyostelium cells are normally not cohesive when grown in shaking suspension in HL5 medium and do not start to adhere to each other until starvation occurs. DdCAD-1 is involved in this initial adhesion (Sesaki \& Siu, 1996). When Ax2::MB and Ax2::MB-rasG(G12T) cells were grown in shaking suspension in the presence of $5 \mu \mathrm{g}$ tetracycline $\mathrm{ml}^{-1}$, they grew as individual cells and were not cohesive (Fig. 2). In contrast, when Ax2: : MB-rasG(G12T) cells were grown in the absence of tetracycline to allow the induction of RasG(G12T) expression, some cells formed clumps, whereas, $\mathrm{Ax} 2: \mathrm{MB}$ cells grown in the absence of tetracycline did not clump (Fig. 2). These results showed that RasG(G12T) expression, rather than simply the removal 

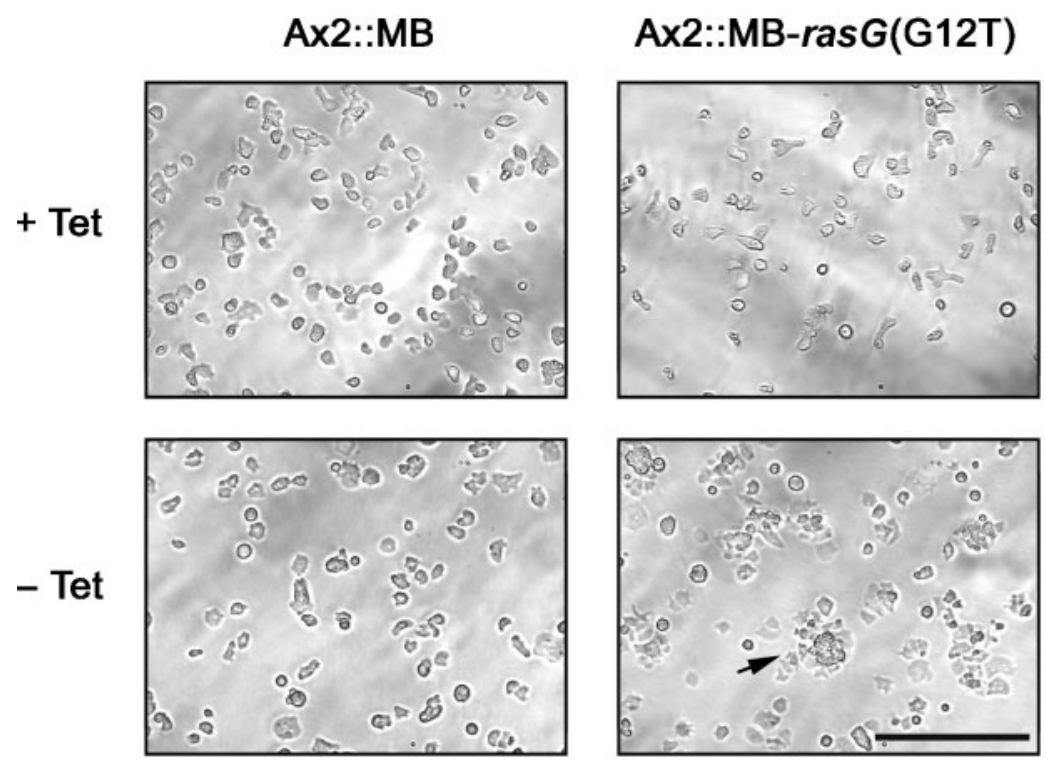

Fig. 2. Induction of cell cohesion during vegetative growth in response to $\operatorname{RasG}(\mathrm{G} 12 \mathrm{~T})$ expression. $\mathrm{A} 2 \mathrm{2}: \mathrm{MB}$ and $\mathrm{A} \times 2: \mathrm{MB}-$ rasG(G12T) cells, grown in HL5 medium in the presence of $5 \mu \mathrm{g}$ tetracycline $\mathrm{ml}^{-1}$ (+Tet) or the absence of tetracycline (-Tet) for $3 \mathrm{~h}$, were photographed through an Olympus CK inverted microscope. The arrow indicates an example of the cell-to-cell cohesion observed in the -Tet Ax2::MB$\operatorname{ras} G(\mathrm{G} 12 \mathrm{~T})$ cell cultures. Bar, $100 \mu \mathrm{m}$. of tetracycline, induced cell-to-cell adhesion. The finding that RasG(G12T) expression was associated with cell cohesion in vegetative cells suggested the possibility that the activation of RasG signalling prematurely induced cell adhesion through the dephosphorylation of DdCAD-1.

To test whether the RasG(G12T)-induced cell clumping was mediated by DdCAD-1, two properties of the adhesion were examined. DdCAD-1 adhesion is inhibited by calcium chelators like EDTA (Wong et al., 1996) and by DdCAD-1specific antibodies (Brar \& Siu, 1993). Ax2::MB and $\mathrm{Ax} 2: \mathrm{MB}-\operatorname{ras} G(\mathrm{G} 12 \mathrm{~T})$ cells were grown for $3 \mathrm{~h}$ in the $\mathrm{ab}-$ sence of tetracycline to allow the induction of $\operatorname{RasG}(\mathrm{G} 12 \mathrm{~T})$, and in the presence of either $10 \mathrm{mM}$ EDTA or $50 \mu \mathrm{g}$ DdCAD-1 antibodies $\mathrm{ml}^{-1}$. The addition of EDTA or the DdCAD-1 antibodies prevented Ax2::MB-rasG(G12T) cells from adhering (Fig. 3), whereas cells incubated in the absence of EDTA or antibody formed clumps as before. These results are consistent with the idea that the observed vegetative cell-to-cell adhesion induced by RasG(G12T) expression is mediated by DdCAD-1.

\section{DdCAD-1 is localized at the cell surface in response to the expression of $\operatorname{RasG(G12T)}$}

During vegetative growth of wild-type Dictyostelium cells in axenic medium, DdCAD-1 is localized in the cytoplasm and the absence of DdCAD-1 at the cell surface may explain why vegetative cells are not adhesive (Sesaki \& Siu, 1996; Sesaki et al., 1997). During development, as cells become more adhesive, some DdCAD-1 becomes localized at the cell surface (Sesaki \& Siu, 1996; Sesaki et al., 1997). The observation that cells expressing RasG(G12T) tended to be adhesive during vegetative growth raised the possibility that DdCAD-1 was localized at the surface upon expression of activated RasG.

Indirect immunofluorescence microscopy was used with DdCAD-1-specific antibodies (Sesaki \& Siu, 1996) to determine if DdCAD-1 was localized at the surface of induced Ax $2:$ MB-rasG(G12T) vegetative cells (Fig. 4). To restrict the staining to DdCAD-1 localized at the cell surface, indirect immunofluorescence microscopy was undertaken using DdCAD-1 antibodies with non-permeabilized cells. Under these conditions, vegetative $\mathrm{Ax} 2: \mathrm{MB}$ cells were largely unstained (Fig. 4), consistent with the finding that DdCAD-1 is localized predominately in the cytoplasm in these cells (Sesaki \& Siu, 1996). In contrast, strongly stained immunofluorescence structures that often formed rings (Fig. 4, arrows) were observed for the vegetative Ax2::MBras $G(\mathrm{G} 12 \mathrm{~T})$ cells, confirming a surface localization of DdCAD-1. Thus, the expression of RasG(G12T) resulted in the movement of some of the DdCAD-1 to the cell surface, providing an explanation as to why the vegetative $\mathrm{Ax} 2: \mathrm{MB}-\operatorname{ras} G(\mathrm{G} 12 \mathrm{~T})$ cells were more cohesive than the control cells.

\section{Cell adhesion during early development is enhanced in RasG(G12T)-expressing cells}

The kinetics of cell cohesion for Ax2 : : MB-rasG(G12T) and Ax $2:: M B$ cells were determined using a standard cell cohesion assay (Lam et al., 1981; Wong et al., 2002). Cells were washed free of tetracycline and starved for $4 \mathrm{~h}$, a time when only EDTA-sensitive DdCAD-1-dependent cell adhesion is occurring (Wong et al., 2002). The cells were dissociated by vigorous vortexing to break up existing aggregates and then allowed to reassociate in rolling tubes (Wong et al., 2002). Some reassociation of the Ax2::MB cells was detected after $20 \mathrm{~min}$ (Fig. 5). The reassociation of induced Ax $2:$ MB-ras $G(\mathrm{G} 12 \mathrm{~T})$ cells was much more rapid, with significant adhesion occurring within 5 min (Fig. 5). The addition of $10 \mathrm{mM}$ EDTA during the assay completely prevented reassociation of both sets of cells, indicating that the observed reassociation was in fact due to EDTA-sensitive adhesion (Fig. 5). These results indicate that cells expressing 


\section{Ax2::MB}

(A)

(B)
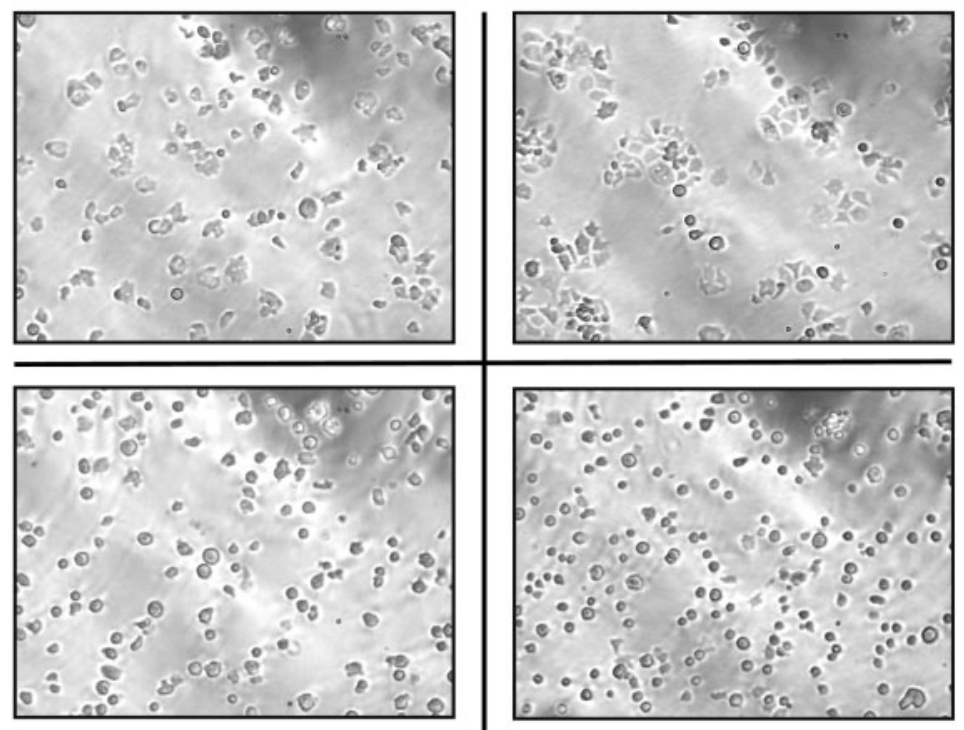

(C)

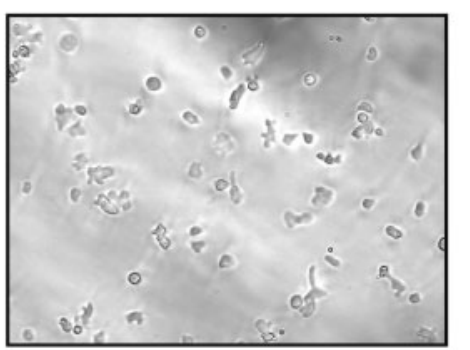

Ax2::MB$\operatorname{ras} G(\mathrm{G} 12 \mathrm{~T})$
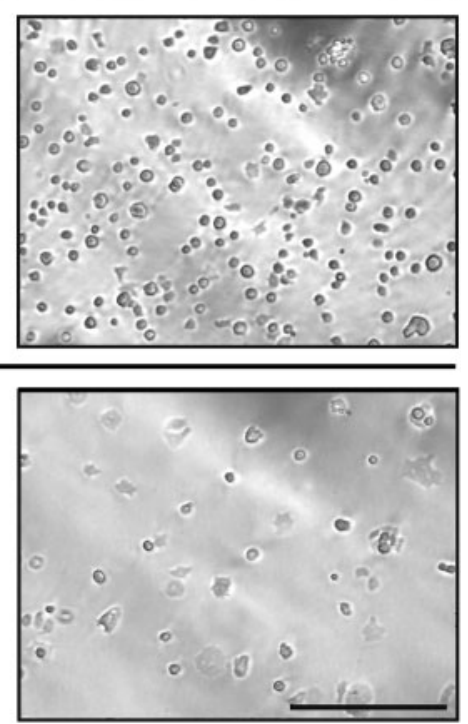

Fig. 3. Disruption of cell cohesion with inhibitors of DdCAD-1-mediated adhesion. Ax2::MB and Ax2::MB-rasG(G12T) cells were washed and incubated for $3 \mathrm{~h}$ in tetracycline-free medium with no addition (A), $1 \mathrm{mM}$ EDTA (B) or $50 \mu \mathrm{g}$ anti-DdCAD1 antibodies $\mathrm{ml}^{-1}$ (C). The cells were placed on Nunc tissue culture dishes, viewed using an Olympus CK inverted microscope and photographed. Bar, $100 \mu \mathrm{m}$.
RasG(G12T) exhibit enhanced DdCAD-1-mediated adhesion during early development.

\section{DdCAD-1 phosphorylation is decreased during Dictyostelium development}

Since cells expressing activated RasG were more cohesive and exhibited decreased DdCAD-1 phosphorylation, there could be an inverse relationship between DdCAD-1 phosphorylation and DdCAD-1-mediated cell cohesion. Since wild-type cells exhibit increased DdCad-1-mediated cell adhesion as development proceeds (Sesaki \& Siu, 1996; Sesaki et al., 1997) and adhesion appeared to be related to phosphorylation, we determined whether DdCAD-1 phosphorylation decreased during development. We used a 1D SDS-PAGE analysis to facilitate the monitoring of multiple samples and to allow a kinetic analysis. cadA null cells were included in the analysis as a control for other phosphothreonine-containing proteins that might co-migrate with DdCAD-1 in the 1D fractionation. A low intensity band, which increased during the development of the cadA null cells, was found to migrate in the same position as

\section{Ax2::MB}

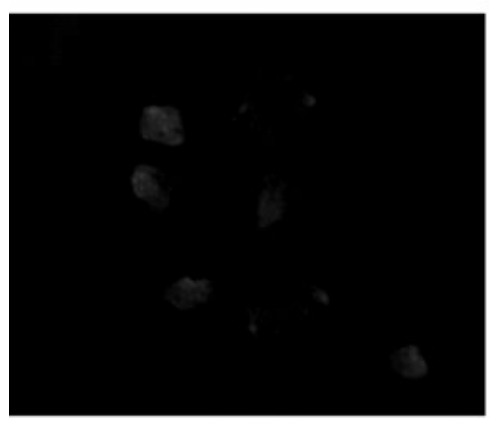

Ax2::MB-rasG(G12T)

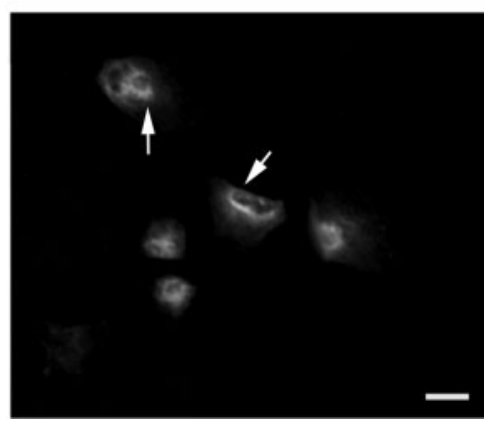

Fig. 4. Immunofluorescent localization of DdCAD-1. Ax2::MB and Ax2::MB-rasG(G12T) cells were grown in the absence of tetracycline for $3 \mathrm{~h}$. Cells were then fixed without permeabilization before being immunostained by indirect immunofluorescence with anti-DdCAD-1 antibodies as described in Methods. The cells were viewed using a Zeiss Axioplan fluorescent microscope and photographed. Bar, $5 \mu \mathrm{m}$. 


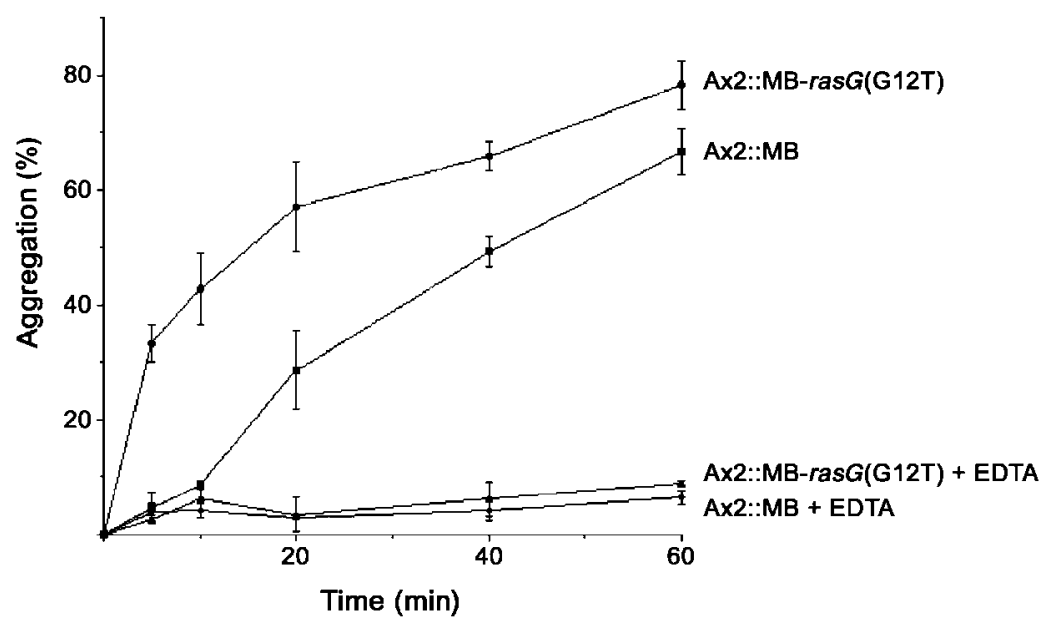

Fig. 5. DdCAD-1 dependent cohesion of developmental cells. Ax2::MB and Ax2::MB$\operatorname{ras} G(\mathrm{G} 12 \mathrm{~T})$ cells were washed free of tetracycline and starved for $4 \mathrm{~h}$ in KK2 in a shaking suspension. Cells were then dissociated by vortexing, and cell reassociation was monitored over time with or without the addition of $10 \mathrm{mM}$ EDTA. Cells were not in aggregates at time zero and the percentage of cell reassociation was calculated by scoring non-aggregated cells with a haemocytometer [percentage aggregation $=($ total number of cells-non-aggregating cells)/total number of cells]. Data represent the mean of three independent experiments $\pm \mathrm{SD}$.

DdCAD-1 (Fig. 6A) and the relative densities of the bands were therefore quantified by densitometry so that the contribution of the non-DdCAD-1 signal could be subtracted. There were relatively constant levels of phosphorylated DdCAD-1 during the first $4 \mathrm{~h}$ of development of the Ax2::MB cells and then levels decreased about fourfold after 6 h (Fig. 6B).

\section{RasG is required for maximum expression of DdCAD-1}

Since the expression of constitutively activated RasG resulted in decreased phosphorylation of DdCAD-1, we postulated that increased phosphorylation of DdCAD-1, and hence reduced DdCAD-1-mediated cohesion, might occur in ras $G$ null ( $r a s G^{-}$) cells. The level of DdCAD-1 phosphorylation was therefore measured in vegetative ras $^{-}$ cells and compared to the level in the parental cells (Fig. 7). Total protein samples, taken from the ras $G^{-}$strain and the control Ax2 strain, were subjected to 2D immunoblot analysis. All three DdCAD-1 phosphoproteins were found in the Ax2 strain as before, but there was a greatly reduced level of phosphorylation in all three DdCAD-1 species in the ras $G^{-}$strain (Fig. 7), rather than the anticipated increase. However, the total amount of DdCAD-1 was greatly decreased in $\mathrm{ras}^{-}$cells in comparison to Ax2 control cells (Fig. 7B). These results indicate that the decrease in DdCAD-1 phosphorylation in ras $G$ null cells is due to a
(A)
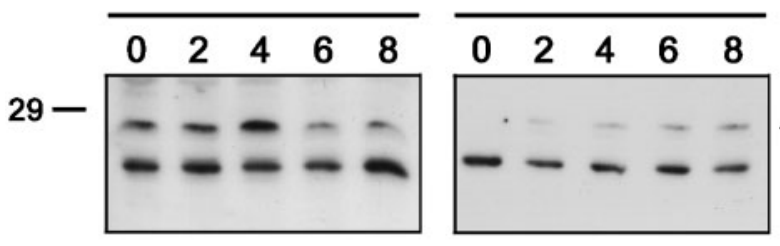

(h)
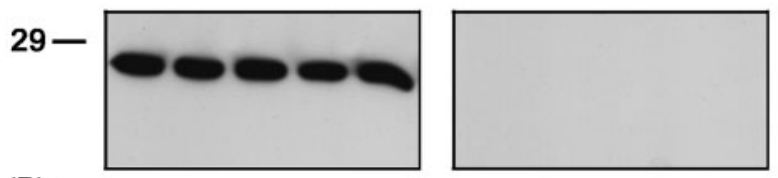

(B)

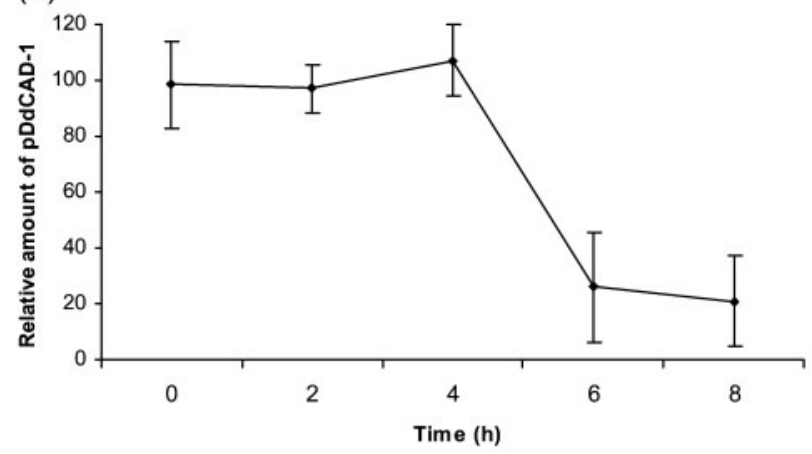

Fig. 6. Levels of DdCAD-1 phosphorylation during development. $\mathrm{Ax} 2: \mathrm{MB}$ and $\mathrm{cadA}^{-}$ cells were starved in $\mathrm{KK} 2$ in a shaking suspension in the absence of tetracycline. (A) At the indicated times, protein samples were fractionated by $1 \mathrm{D}$ electrophoresis and immunoblots were probed with anti-phosphothreonine antibodies to detect phosphorylated DdCAD-1. The filled arrowhead shows the position of phosphorylated DdCAD-1. Blots were then stripped of bound antibody and reprobed with anti-DdCAD-1 antibodies to detect total DdCAD-1 levels (open arrow). Molecular mass $(\mathrm{kDa})$ markers are indicated. (B) The immunoblots probed with anti-phosphothreonine antibodies shown in (A) were quantified by densitometric analysis. The non-DdCAD-1 phosphothreonine-stained material that cofractionated with DdCAD-1 in the $\operatorname{cad} A$ null cells was subtracted from the values for $\mathrm{Ax} 2: \mathrm{MB}$ and normalized relative to amount of phosphorylated DdCAD-1 in Ax2::MB cells at $O \mathrm{~h}$. Data from three independent experiments are shown. 
(A)

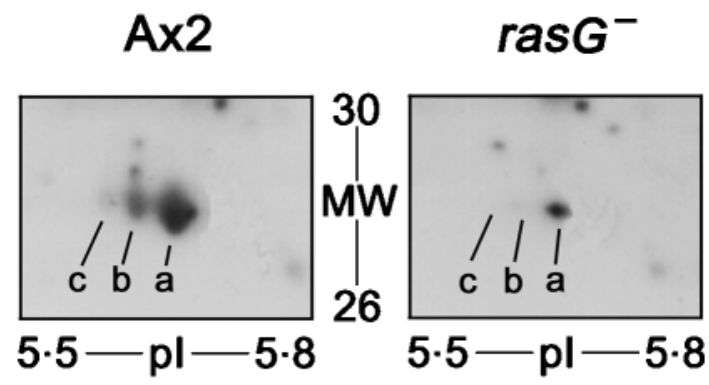

(B)

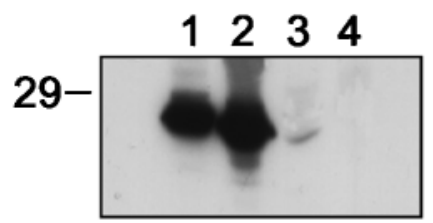

Fig. 7. Levels of total and phosphorylated DdCAD-1 in a rasG null and Ax2 control cells. (A) Total protein from a ras $G^{-}$strain and $A \times 2$ was fractionated by $2 D$ electrophoresis. Blotted membranes were probed with anti-phosphothreonine antibody to detect phosphoproteins. The section of the 2D immunoblot blot known to contain DdCAD-1 is shown ( $\mathrm{a}=\mathrm{DdCAD}-1 \mathrm{a}$, $b=$ DdCAD-1b, $c=$ DdCAD-1c). (B) Total protein from induced $A \times 2:: M B$ (lane 1), induced $A \times 2:: M B-r a s G(G 12 T)$ (lane 2), ras $G^{-}$(lane 3 ) and $\operatorname{cadA}^{-}$(lane 4) cells were fractionated by 1D electrophoresis and blotted with a DdCAD-1-specific antibody. Molecular mass $(\mathrm{kDa})$ markers and $\mathrm{pl}$ ranges are indicated.

decrease in DdCAD-1 protein level, suggesting that RasG is an important regulator of DdCAD-1 expression during vegetative growth.

\section{DISCUSSION}

In this study, we have confirmed that the phosphorylated molecules, tentatively identified as DdCAD-1 species (Secko et al., 2004), are in fact DdCAD-1 since they are absent in a cadA null cell line. Although DdCAD-1 has long been implicated as a major adhesion molecule during early development (Coates \& Harwood, 2001), its phosphorylation has not been previously described. Since DdCAD-1 was resolved into at least three spots by $2 \mathrm{D}$ electrophoresis, it is likely that it is phosphorylated at more than one site. In addition, since the separated components were all detected with a phosphothreonine-specific antibody, each must contain a phosphorylated threonine residue, although the pI shift might be due to modification of other amino acid residues. Since total DdCAD-1 protein levels were not significantly different between cells expressing RasG(G12T) and control cells (Fig. 1B), the changes in DdCAD-1 phosphorylation were not a result of changes in protein level. These results suggest the existence of phosphatases and/or kinases that act on DdCAD-1, which are also among the downstream effectors of RasG.

DdCAD-1-mediated adhesion is known to be homophilic (i.e. binding is through DdCAD-1/DdCAD-1 interactions) and requires $\mathrm{Ca}^{2+}$ (Brar \& Siu, 1993; Wong et al., 1996). The work reported here suggests that DdCAD-1 is also regulated by phosphorylation. Initial insight into the effect of phosphorylation on DdCAD-1 function came from the observation that cells expressing RasG(G12T) became cohesive during vegetative growth (Fig. 5). Constitutive gp 80 overexpression in vegetative cells has been found to induce EDTA-stable contacts in suspension (Faix et al., 1992). However, the RasG(G12T)-induced cohesion was EDTAsensitive and was blocked with DdCAD-1 antibodies, indicating that it was mediated by DdCAD-1. The fact that the $\operatorname{RasG}(\mathrm{G} 12 \mathrm{~T})$-expressing cells were more cohesive and contained less phosphorylated DdCAD-1 suggested the possibility that dephosphorylation of DdCAD-1 was important in facilitating DdCAD-1-mediated adherence. An important regulatory role for phosphorylation in mammalian cell adhesion has been documented (Lickert et al., 2000; Lilien et al., 2002; Nelson \& Nusse, 2004), but this is the first time such a role has been implicated in Dictyostelium cell adhesion.

Bacterially grown cells do not express DdCAD-1 until the onset of starvation (Knecht et al., 1987), but axenically grown vegetative cells express high levels of DdCAD-1. These latter cells are not adhesive, because the protein is sequestered in the cytoplasm (Brar \& Siu, 1993; Sesaki \& Siu, 1996). Axenically grown cells only became adhesive after the onset of starvation and this correlated with the movement of DdCAD-1 to the cell surface. In contrast, axenically grown, $\operatorname{RasG}(\mathrm{G} 12 \mathrm{~T})$-expressing vegetative cells were already cohesive and this correlated with the presence of DdCAD-1 at the cell surface in ring-like structures (Fig. 4), suggesting that a $\operatorname{RasG}(\mathrm{G} 12 \mathrm{~T})$-dependent signal regulated the movement of DdCAD-1.

Transport of DdCAD-1 to the cell surface has been shown to occur via a non-classical pathway involving contractile vacuoles (Sesaki et al., 1997). Sesaki et al. (1997) have suggested that this occurs by at least four steps, including (1) DdCAD-1 accumulation into contractile vacuoles, (2) the binding of DdCAD-1 to the contractile vacuole membrane, (3) the association of DdCAD-1 with an 'anchoring' protein on the luminal surface of the vacuoles, and (4) the fusion of a contractile vacuole with the plasma membrane followed by the subsequent dispersal of DdCAD-1 across the surface of the cell. Our data suggest that RasG(G12T) may affect this transport pathway. This possibility is supported by the fact that, after $3 \mathrm{~h}$ of development, DdCAD-1-filled contractile vacuoles appear as ring-like structures when merging with the plasma membrane (Sesaki et al., 1997). These ring-like structures bear a distinct similarity to the immunofluorescence staining in $\operatorname{RasG}(\mathrm{G} 12 \mathrm{~T})$-expressing vegetative cells, 
which is often concentrated into rings (Fig. 4, arrows). Since these structures are not seen until $3 \mathrm{~h}$ of development in wild-type cells (Sesaki et al., 1997), this further suggests that $\operatorname{RasG}(\mathrm{G} 12 \mathrm{~T})$ is prematurely activating this pathway. However, it has yet to be established what role phosphorylation may play in this process and, as such, it is not yet clear whether the phosphorylation of DdCAD-1 regulates its localization or, conversely, whether the localization of DdCAD-1 regulates its phosphorylation.

We previously found that ras $G$ null cells express lower levels of discoidin, a protein normally induced at the onset of development (Secko et al., 2001). We have now demonstrated that ras $G$ null cells have considerably lower levels of the cell adhesion protein DdCAD-1 (Fig. 7). This observation came as something of a surprise based on the fact that the expression of RasG(G12T) did not affect DdCAD-1 protein levels under the short-term induction conditions used here. However, the observed decrease in total levels of DdCAD-1 was much larger than the decrease observed for DdCAD-1 phosphorylation in these cells (see Fig. 7A versus $7 \mathrm{~B}$ ), which argues that, at least qualitatively, DdCAD-1 is more phosphorylated in the ras $G$ null cells. The decrease in levels of both discoidin and DdCAD-1 in rasG null cells also suggests that RasG signalling may play a role in protein expression during early development.

DdCAD-1 shares limited homology with the extracellular domain of metazoan cadherins; in particular, the two middle domains of DdCAD-1 (aa 49-146) share $28 \%$ identity with the first extracellular repeat of E-cadherin, as well as similarities in their $\mathrm{Ca}^{2+}$-binding site (Wong et al., 1996). Cadherins are involved in cell-cell adhesion and the formation of adherence junctions (Nelson \& Nusse, 2004). Metazoan cadherins form complexes with catenins to allow their linkage to the actin cytoskeleton (Jamora \& Fuchs, 2002) and the stability of these complexes is regulated by phosphorylation. Tyrosine phosphorylation of $\beta$-catenin by Src or Fer results in the disruption of cadherin/ $\beta$-catenin complexes and decreased adhesion (Lilien et al., 2002; Nelson \& Nusse, 2004). An unidentified transmembrane linker has been postulated to bind to DdCAD-1 (Sesaki et al., 1997); however, the proteins that interact with DdCAD-1 are currently not known. It is possible that DdCAD-1 phosphorylation performs a similar function. It should be noted that cadherin $/ \beta$-catenin complexes are phosphorylated on serine/threonine residues, which results in increased stabilization of the complexes (Lickert et al., 2000). Since DdCAD1 also appears to be phosphorylated on several residues (Fig. 1), its regulation by phosphorylation could be equally complex. It must also be pointed out that DdCAD-1 is not a transmembrane protein and thus its mode of adhesion could therefore be distinct from systems described in mammalian cells. Nonetheless, it will be of interest to determine the pathway leading from RasG activation to DdCAD-1 dephosphorylation, as this will not only help in our understanding of Dictyostelium development, but could produce insights into the regulation of metazoan cell-cell adhesion.

\section{ACKNOWLEDGEMENTS}

This research was supported by a Canadian Institutes of Health Research Operating Grant to G. W.

\section{REFERENCES}

Beug, H., Katz, F. E., Stein, A. \& Gerisch, G. (1973). Quantitation of membrane sites in aggregating Dictyostelium cells by use of tritiated univalent antibody. Proc Natl Acad Sci U S A 70, 3150-3154.

Brar, S. K. \& Siu, C. H. (1993). Characterization of the cell adhesion molecule gp24 in Dictyostelium discoideum. Mediation of cell-cell adhesion via a $\mathrm{Ca}^{2+}$-dependent mechanism. J Biol Chem 268, 24902-24909.

Choi, A. H. \& Siu, C. H. (1987). Filopodia are enriched in a cell cohesion molecule of $\mathrm{Mr} 80,000$ and participate in cell-cell contact formation in Dictyostelium discoideum. J Cell Biol 104, 1375-1387.

Coates, J. C. \& Harwood, A. J. (2001). Cell-cell adhesion and signal transduction during Dictyostelium development. J Cell Sci 114, 43494358.

Faix, J., Gerisch, G. \& Noegel, A. A. (1992). Overexpression of the csA cell adhesion molecule under its own cAMP-regulated promoter impairs morphogenesis in Dictyostelium. J Cell Sci 102, 203-214.

Fukui, Y., Yumura, S. \& Yumura-Kitanishi, T. (1987). Agar-overlay immuno-fluorescence: high resolution studies of cytoskeletal components and their changes during chemotaxis. Methods Cell Biol 28 , 347-356.

Gerisch, G. (1968). Cell aggregation and differentiation in Dictyostelium. Curr Top Dev Biol 3, 157-197.

Jamora, C. \& Fuchs, E. (2002). Intercellular adhesion, signalling and the cytoskeleton. Nat Cell Biol 4, E101-E108.

Juliano, R. L. (2002). Signal transduction by cell adhesion receptors and the cytoskeleton: functions of integrins, cadherins, selectins, and immunoglobulin-superfamily members. Annu Rev Pharmacol Toxicol 42, 283-323.

Kae, H., Lim, C. J., Spiegelman, G. B. \& Weeks, G. (2004). Chemoattractant-induced Ras activation during Dictyostelium aggregation. EMBO Rep 5, 602-606.

Kessin, R. H. (2001). Dictyostelium - Evolution, Cell Biology, and the Development of Multicellularity. Cambridge: Cambridge University Press.

Khosla, M., Spiegelman, G. B., Weeks, G., Sands, T. W., Virdy, K. J. \& Cotter, D. A. (1994). RasG protein accumulation occurs just prior to amoebae emergence during spore germination in Dictyostelium discoideum. FEMS Microbiol Lett 117, 293-298.

Khosla, M., Spiegelman, G. B. \& Weeks, G. (1996). Overexpression of an activated ras $G$ gene during growth blocks the initiation of Dictyostelium development. Mol Cell Biol 16, 4156-4162.

Knecht, D. A., Fuller, D. L. \& Loomis, W. F. (1987). Surface glycoprotein, gp24, involved in early adhesion of Dictyostelium discoideum. Dev Biol 121, 277-283.

Lam, T. Y., Pickering, G., Geltosky, J. \& Siu, C. H. (1981). Differential cell cohesiveness expressed by prespore and prestalk cells of Dictyostelium discoideum. Differentiation 20, 22-28.

Lickert, H., Bauer, A., Kemler, R. \& Stappert, J. (2000). Casein kinase II phosphorylation of E-cadherin increases E-cadherin/ $\beta$-catenin interaction and strengthens cell-cell adhesion. J Biol Chem 275, 5090-5095.

Lilien, J., Balsamo, J., Arregui, C. \& Xu, G. (2002). Turn-off, dropout: functional state switching of cadherins. Dev Dyn 224, 18-29.

Lim, C. J., Spiegelman, G. B. \& Weeks, G. (2001). RasC is required for optimal activation of adenylyl cyclase and Akt/PKB during aggregation. EMBO J 20, 4490-4499. 
Muller, K. \& Gerisch, G. (1978). A specific glycoprotein as the target site of adhesion blocking $\mathrm{Fab}$ in aggregating Dictyostelium cells. Nature 274, 445-449.

Nelson, W. J. \& Nusse, R. (2004). Convergence of Wnt, beta-catenin, and cadherin pathways. Science 303, 1483-1487.

Primpke, G., lassonidou, V., Nellen, W. \& Wetterauer, B. (2000). Role of cAMP-dependent protein kinase during growth and early development of Dictyostelium discoideum. Dev Biol 221, 101-111.

Secko, D. M., Khosla, M., Gaudet, P., Tsang, A., Spiegelman, G. B. \& Weeks, G. (2001). RasG regulates discoidin gene expression during Dictyostelium growth. Exp Cell Res 266, 135-141.

Secko, D. M., Insall, R. H., Spiegelman, G. B. \& Weeks, G. (2004). The identification of Dictyostelium phosphoproteins altered in response to the activation of RasG. Proteomics 4, 2629-2639.

Sesaki, H. \& Siu, C. H. (1996). Novel redistribution of the $\mathrm{Ca}^{2+}$. dependent cell adhesion molecule DdCAD-1 during development of Dictyostelium discoideum. Dev Biol 177, 504-516.

Sesaki, H., Wong, E. F. \& Siu, C. H. (1997). The cell adhesion molecule DdCAD-1 in Dictyostelium is targeted to the cell surface by a nonclassical transport pathway involving contractile vacuoles. J Cell Biol 138, 939-951.

Tuxworth, R. I., Cheetham, J. L., Machesky, L. M., Spiegelmann, G. B., Weeks, G. \& Insall, R. H. (1997). Dictyostelium RasG is required for normal motility and cytokinesis, but not growth. J Cell Biol 138, 605-614.

Watts, D. \& Ashworth, J. (1970). Growth of myxamoebae of the cellular slime mould Dictyostelium discoideum in axenic culture. Biochem J 119, 171-174.

Weeks, G. \& Spiegelman, G. B. (2003). Roles played by Ras subfamily proteins in the cell and developmental biology of microorganisms. Cell Signal 15, 901-909.

Wong, E. F., Brar, S. K., Sesaki, H., Yang, C. \& Siu, C. H. (1996). Molecular cloning and characterization of DdCAD-1, a $\mathrm{Ca}^{2+}$ dependent cell-cell adhesion molecule, in Dictyostelium discoideum. J Biol Chem 271, 16399-16408.

Wong, E., Yang, C., Wang, J., Fuller, D., Loomis, W. F. \& Siu, C. H. (2002). Disruption of the gene encoding the cell adhesion molecule DdCAD-1 leads to aberrant cell sorting and cell-type proportioning during Dictyostelium development. Development 129, 3839-3850.

Yang, C., Brar, S. K., Desbarats, L. \& Siu, C. H. (1997). Synthesis of the $\mathrm{Ca}^{2+}$-dependent cell adhesion molecule DdCAD-1 is regulated by multiple factors during Dictyostelium development. Differentiation 61, 275-284.

Zeng, C., Anjard, C., Riemann, K., Konzok, A. \& Nellen, W. (2000). gdt1, a new signal transduction component for negative regulation of the growth-differentiation transition in Dictyostelium discoideum. Mol Biol Cell 11, 1631-1643. 